Article

\title{
Freeform Fabrication of Magnetophotonic Crystals with Diamond Lattices of Oxide and Metallic Glasses for Terahertz Wave Control by Micro Patterning Stereolithography and Low Temperature Sintering
}

\author{
Soshu Kirihara * and Maasa Nakano \\ Joining and Welding Research Institute, Osaka University, 11-1 Mihogaoka Ibaraki, 567-0047 Osaka, \\ Japan; E-Mail: nakano@jwri.osaka-u.ac.jp \\ * Author to whom correspondence should be addressed; E-Mail: kirihara@jwri.osaka-u.ac.jp; \\ Tel.: +81-6-6879-8693; Fax: +81-6-6879-8693.
}

Received: 25 January 2013; in revised form: 26 March 2013 / Accepted: 26 March 2013 /

Published: 2 April 2013

\begin{abstract}
Micrometer order magnetophotonic crystals with periodic arranged metallic glass and oxide glass composite materials were fabricated by stereolithographic method to reflect electromagnetic waves in terahertz frequency ranges through Bragg diffraction. In the fabrication process, the photo sensitive acrylic resin paste mixed with micrometer sized metallic glass of $\mathrm{Fe}_{72} \mathrm{~B}_{14.4} \mathrm{Si}_{9.6} \mathrm{Nb}_{4}$ and oxide glass of $\mathrm{B}_{2} \mathrm{O}_{3} \cdot \mathrm{Bi}_{2} \mathrm{O}_{3}$ particles was spread on a metal substrate, and cross sectional images of ultra violet ray were exposed. Through the layer by layer stacking, micro lattice structures with a diamond type periodic arrangement were successfully formed. The composite structures could be obtained through the dewaxing and sintering process with the lower temperature under the transition point of metallic glass. Transmission spectra of the terahertz waves through the magnetophotonic crystals were measured by using a terahertz time domain spectroscopy.
\end{abstract}

Keywords: metallic and oxide glass composite; magnetophotonic crystals; terahertz wave; micro stereolithography

\section{Introduction}

Magnetophotonic crystals with periodic arrangements of magnetic and dielectric materials can form forbidden bands to totally reflect electromagnetic waves with wavelengths comparable to the lattice 
constant though Bragg diffraction [1]. The created forbidden regions in the transmission spectra are called especially photonic band gap. Through introductions of air holes as the structural defects into the periodic arrangement, transmission peaks of localized modes will be formed at the specific frequencies according to the cavity sizes [2]. In our previous research, fine alumina or zirconia lattices with diamond structures were developed to control microwaves by using a stereolithography of computer aided designing and manufacturing [3-5]. Recently, the electromagnetic waves in terahertz frequency ranges were verified to synchronize with collective vibration modes of various harmful substances. The spectroscopic technologies are expected to be applied to sensors for real time detecting toxic materials $[6,7]$. However, the terahertz waves are difficult to transmit into the water solvents due to electromagnetic absorptions [8]. Novel resonators to concentrate the electromagnetic wave energies are required strongly. In this investigation, the micrometer order magnetophotonic crystals with the diamond lattice structure composed of metallic glass of $\mathrm{Fe}_{72} \mathrm{~B}_{14.4} \mathrm{Si}_{9.6} \mathrm{Nb}_{4}$ dispersed oxide glass of $\mathrm{B}_{2} \mathrm{O}_{3} \cdot \mathrm{Bi}_{2} \mathrm{O}_{3}$ were fabricated by the micro stereolithography to control the terahertz waves effectively. The device image is schematically illustrated in Figure 1. The iron based metallic glass is soft magnetic amorphous alloys with high glass forming ability and the magnetic permeability [9]. The artificial crystal having the cylindrical structural defect can resonate with the specific terahertz wave with the wavelength comparable to the cylinder diameter, and the localized mode of transmission peak appears in the band gap. When various water solvents flowed into the micro tube, the localized modes will be shifted in the gap according to the dielectric constants of the liquid materials. If the resonated wave can harmonize with the collective vibration mode of the introduced water solvents, the transmission peak will be disappeared through the electromagnetic absorptions. Moreover, the profiles and frequency ranges of the band gaps can be modulated by the permeability control of the diffraction lattices through applying the static magnetic field. The magnetophotonic crystal sensors will be applied to various scientific and engineering fields by utilizing the terahertz wave spectroscopic database.

Figure 1. A schematic illustrated a magnetophotonic crystal sensor device (a) and transmission spectra of terahertz waves including various water solvents without (b) or with (c) absorption properties.

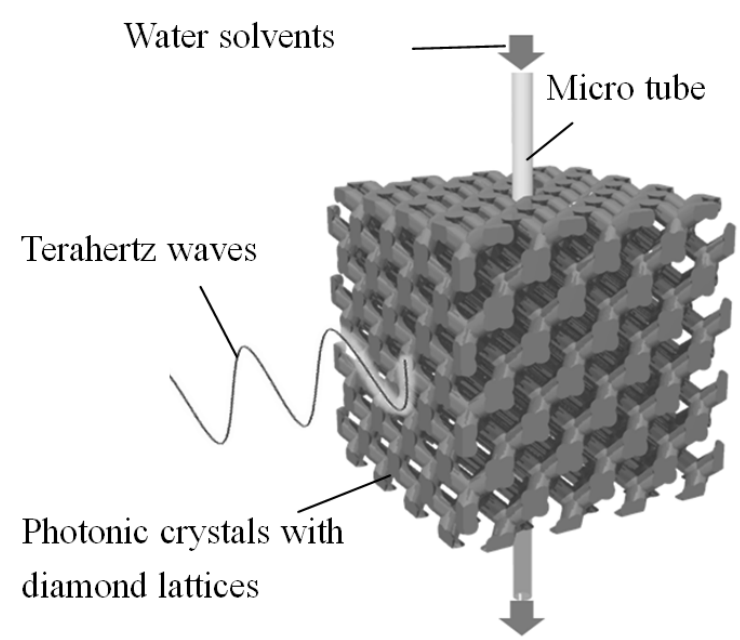

(a)

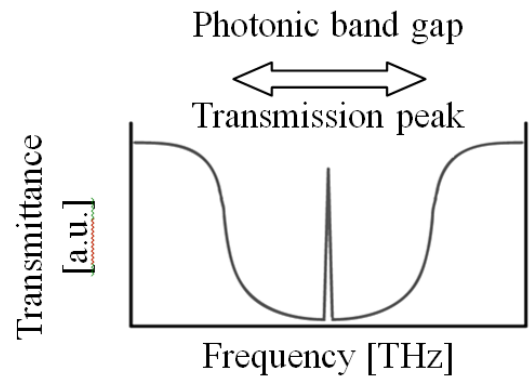

(b)

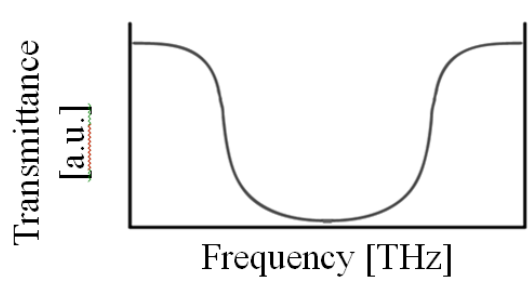

(c) 


\section{Experimental Section}

The micro pattering stereolithography illustrated schematically in Figure 2 was used to fabricate the fine magnetophotonic components. A three dimensional model designed by a computer graphic software (Materialise Japan Co., Ltd., Yokoyama, Japan, Magics Ver. 14) was sliced into a series of two dimensional cross sectional data of $10 \mu \mathrm{m}$ in layer thickness, and these processed data sets were transferred into the stereolithographic equipment (D-MEC Co., Ltd., Tokyo, Japan, SI-C 1000). Photo sensitive acrylic resins dispersed with the metallic glass of $\mathrm{Fe}_{72} \mathrm{~B}_{14.4} \mathrm{Si}_{9.6} \mathrm{Nb}_{4}$ and oxide glass of $\mathrm{B}_{2} \mathrm{O}_{3} \cdot \mathrm{Bi}_{2} \mathrm{O}_{3}$ particles of $2.6 \mu \mathrm{m}$ and $1.0 \mu \mathrm{m}$ in diameters was supplied on a metal substrate and spread uniformly by a mechanical knife edge. The thickness of each layer was controlled to $10 \mu \mathrm{m}$. The two dimensional pattern was exposed clearly by illuminating an ultra violet laser of $405 \mathrm{~nm}$ in wavelength on the slurry surface. The high resolution was achieved by using a digital micro-mirror device and an objective lens. This optical device was composed of squire aluminum mirrors of $14 \mu \mathrm{m}$ in edge length with $1024 \times 768$ in numbers. The each tiny mirror could be tilted independently according to the two dimensional cross sections by the computer operation. Through the layer by layer slurry spreading and pattern exposing, the three dimensional fine structures could be created automatically. The stereolithographic process parameters were optimized to create the magnetophotonic components exactly through exploratory modeling by using the prepared slurries with different compositions. The mixed particles of the metallic and oxide glasses were dispersed into the acrylic resin at $40 \mathrm{vol} . \%$ in total, and the mixed ratios of these particles were varied at 16:24, 17:23 and 18:22 in volume. The model shown in Figure 3 was designed and fabricated to measure the layer thickness of photo polymerization depth and the size tolerance of exposed right scattering. The magnetophotonic crystals with the diamond lattice structure were designed and fabricated along the optimized stereolithographic conditions. The micro rods of 144 and $217 \mu \mathrm{m}$ in diameter and length created the diamond structure of $500 \mu \mathrm{m}$ in lattice constant, respectively. The device component of $5.0 \times 5.0 \times 0.5 \mathrm{~mm}$ in external dimensions was formed through connecting unit cells of $10 \times 10 \times 1$ in numbers. The transition temperature for the crystal phase of the selected metallic glass with the amorphous structure was measured as $552{ }^{\circ} \mathrm{C}$ in the previous research [7-9]. The melting temperature of the selected oxide glass was $450{ }^{\circ} \mathrm{C}$ approximately. The formed precursors were dewaxed at $420{ }^{\circ} \mathrm{C}$ for $8.0 \mathrm{~h}$ with the heating rate of $1.0{ }^{\circ} \mathrm{C} / \mathrm{min}$, and sintered at $460{ }^{\circ} \mathrm{C}$ for $0.5 \mathrm{~h}$ with the heating rate of $2.0{ }^{\circ} \mathrm{C} / \mathrm{min}$ in an $\mathrm{Ar}$ atmosphere. Microstructures of the composite lattice were observed by an optical microscopy and a scanning electron microscopy. The microstructural stabilities of the amorphous phase were analyzed by using an X-ray diffraction. The terahertz wave transmittances through the magnetophotonic crystals were measured and calculated by using a terahertz time domain spectroscopy (Aispec Co., Ltd., Hachioji, Japan, J-spec $2001 \mathrm{spc} / \mathrm{ou}$ ) and a transmission line modeling simulator (CST AG, Darmstadt, Germany, Microstripes, Ver. 12). 
Figure 2. Schematic illustrations of a stereolithographic method. A three dimensional model was sliced into two dimensional cross sections by computer graphic technique (a). A micrometer order component could be created successfully through layer by layer smoothly slurry spreading and finely pattern exposing (b).

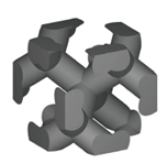

3D graphic model

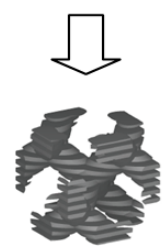

A series of cross sectional data

(a)
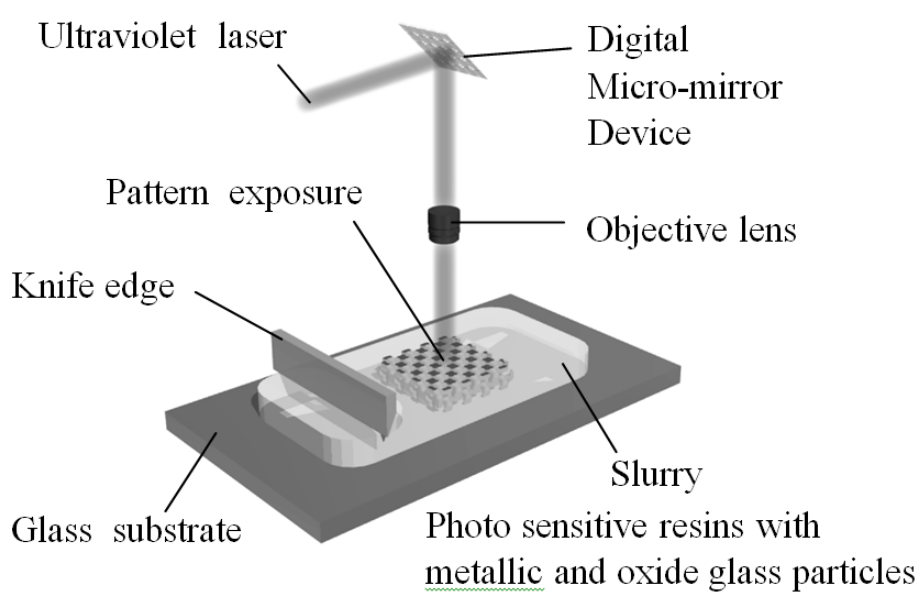

(b)

Figure 3. A graphic model of test specimen to optimize the stereolithographic process parameters (a). Layer thicknesses of photo polymerization depths (b) and size tolerances of exposure right scattering (c) were measured.

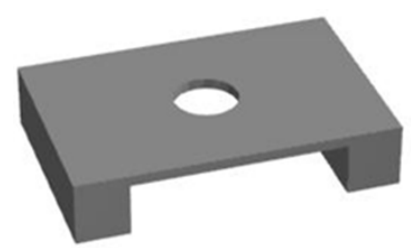

(a)

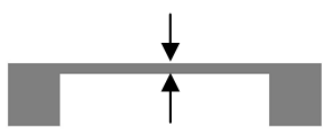

(b)

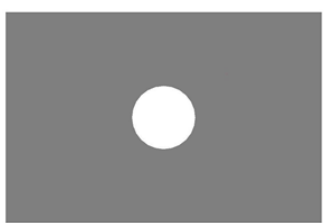

(c)

\section{Results and Discussion}

In the optimization of the stereolithgrapic parameters, the exposure power influences toward the layer thicknesses and size tolerances were compared and investigated systematically in the formed acryl objects with the different mixing ratio of the metallic and oxide glasses. The cross sectional object of 14.5 and $\pm 3 \mu \mathrm{m}$ in layer thickness and size tolerance were created by micro patterning of $700 \mathrm{~mJ} / \mathrm{cm}^{2}$ in exposure power for the mixed slurry composed of the acrylic resin, metallic and oxide glass particles of 60,17 and $23 \%$ in volume fractions, respectively. The photo polymerization depth should be over $10 \mu \mathrm{m}$ of the stacking layer thickness with restraining the exposure right scatters. Figure $4 \mathrm{a}$ shows the magnetophotonic crystals with the diamond lattices fabricated by the stereolithography. The size tolerance between the designed model and formed sample was converged within $\pm 3 \mu \mathrm{m}$. The sintered diamond lattice structure with $500 \mu \mathrm{m}$ in lattice constant is shown in Figure $4 \mathrm{~b}$. The micrometer order periodic lattices were formed successfully. The liner shrinkage ratios of horizontal and vertical axis were $10.2 \%$ and $12.5 \%$, respectively. These results can be fed back successfully for the computer graphic designing to realize the isotropic arrangement of diamond lattice. Figure 5 shows the microstructure of the sintered metallic glass and oxide glass composite 
lattice. The metallic glass particles dispersed homogeneously in the oxide glass matrix. The X-ray diffraction patterns of metallic glass particles before and after the heat treatments were analyzed as shown in Figure 6. The metallic glass was not crystallized during the dewaxing and sintering heat treatments. Figure 7 shows the measured terahertz wave transmission spectrum for the magnetophotonic crystal. The black and gray lines show the measured and calculated results, respectively. These transmission spectra have good agreement. The theoretical visualization of the electromagnetic wave propagation could verify the forbidden gap was exhibited by the wave diffraction. The electromagnetic band gap can be formed from 0.2 to $1.0 \mathrm{THz}$ in wave frequency. Between the lower and higher band gap edges, the electromagnetic waves from 300 to $1500 \mu \mathrm{m}$ in wavelengths create standing vibrations in the periodic arrangements of magnetophotonic lattices and realize the total reflections for the incident direction through the Bragg diffractions. Figure 8 shows the magnetophotonic crystal with a structural defect fabricated by using the micro pattering stereolithography and the low temperature heat treatment. A round hole of $200 \mu \mathrm{m}$ in diameter was opened as the defect cavity exactly into the diamond lattice structure for perpendicularity direction toward the crystal face shown in Figure 8. The terahertz wave was propagated toward the parallel direction to the opened hole, and the transmission spectrum was obtained as shown in Figure 9. The black and gray spectra show the measured and calculated ones, respectively. The localized modes of the transmission peaks were formed in the band gaps. At the peak frequencies of $0.72,0.75$ and $0.79 \mathrm{THz}$, the half wavelengths of 208, 200 and 189 were comparable to the cavity diameter of defect hole, and the standing waves should be formed by multiple reflections between the diffraction lattices. The amplified terahertz waves could be transmitted selectively toward the opposite side of the magnetophotonic crystal. Because the opened hole has uneven surface, the similar wavelengths could resonate in the cavity, and the three localized modes were considered to be formed in the band gap as shown in Figure 9. The electromagnetic fields profiles of these localized modes were calculated and visualized in the cylindrical cavity. Moreover, the intentional shifts of the band gap frequency and localized mode peaks could be simulated through the lattice permeability modulations supposing the static magnetic field application toward the magnetophotonic crystal.

Figure 4. An acryl diamond lattice with metallic glass $\left(\mathrm{Fe}_{72} \mathrm{~B}_{14.4} \mathrm{Si}_{9.6} \mathrm{Nb}_{4}\right)$ and oxide glass $\left(\mathrm{B}_{2} \mathrm{O}_{3} \cdot \mathrm{Bi}_{2} \mathrm{O}_{3}\right)$ particles dispersion fabricated by the stereolithography (a) and a magnetophotonic crystal after dewaxing and sintering (b).

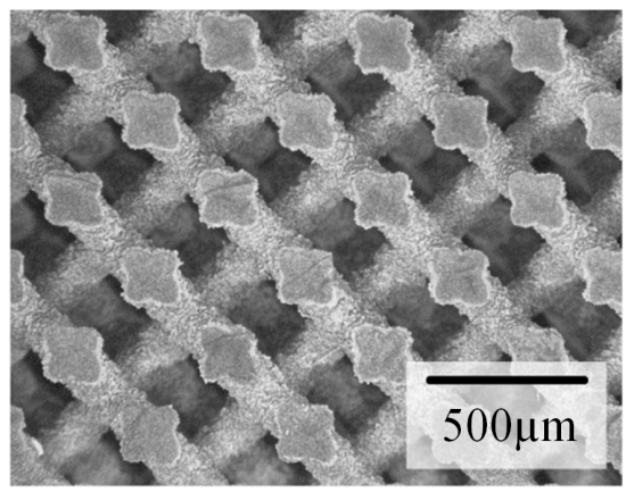

(a)

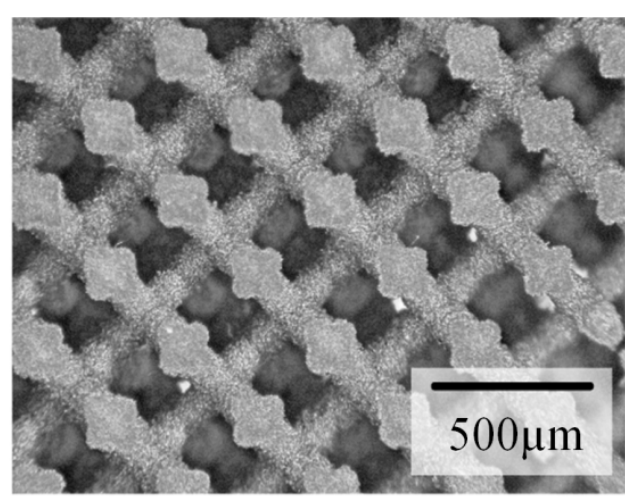

(b) 
Figure 5. A microstructure of the oxide glass lattice with the metallic glass particles dispersion observed by a scanning electron microscopy.

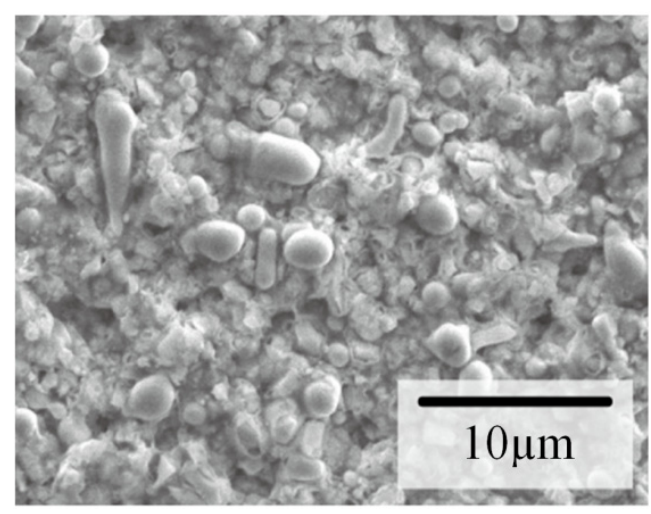

Figure 6. A X-ray diffraction patterns of the metallic glass particles before and after the dewaxing and sintering heat treatments under the transition temperature from the amorphous structure for the crystal phase.

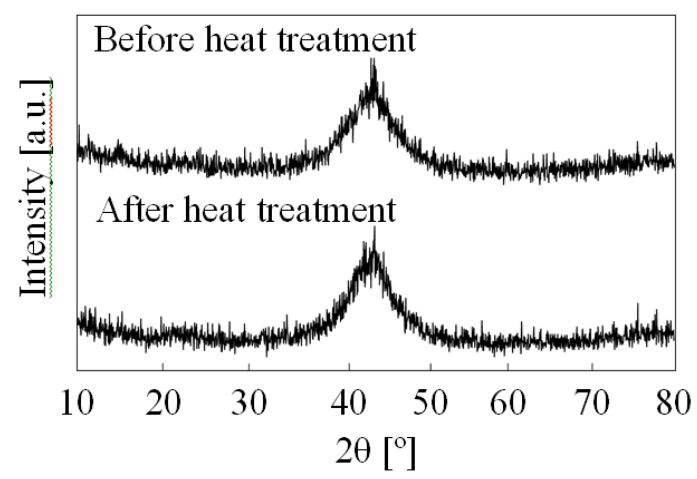

Figure 7. An electromagnetic band gap formation in a transmission spectrum of the terahertz wave through the magnetophotonic crystal with the diamond lattice structure. The black and gray lines show the measured and calculated results, respectively.

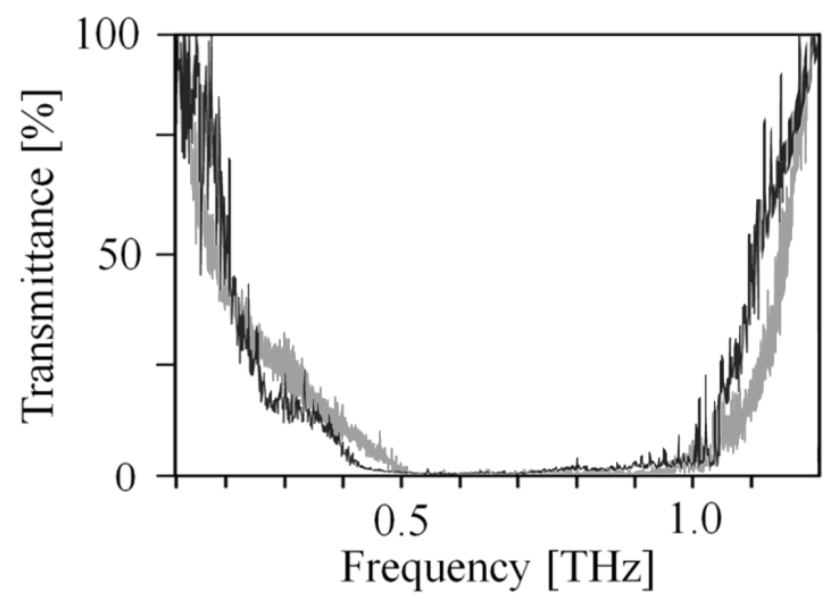


Figure 8. The magnetophotonic crystal with a structural defect in the periodic lattice of the diamond structure. The round hole was opened as the defect cavity for the perpendicularly direction toward the crystal face.

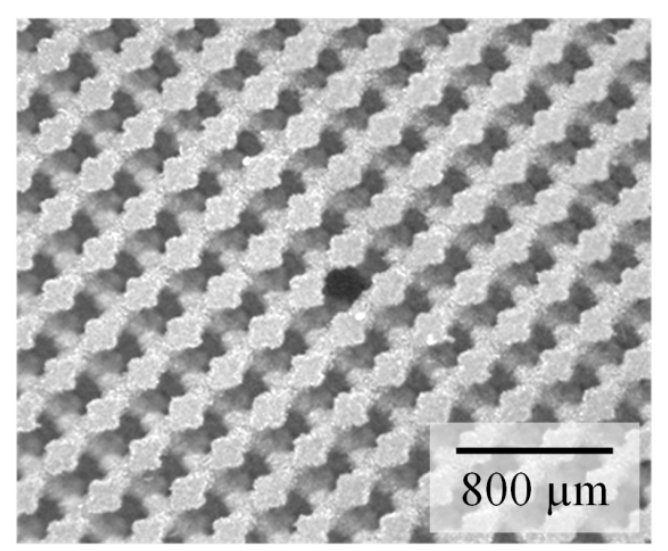

Figure 9. Localized modes formation in the electromagnetic band gap. The terahertz waves with the selected wavelengths can resonate with the defect cavity and transmit the crystal. The black and gray lines show the measured and calculated results, respectively.

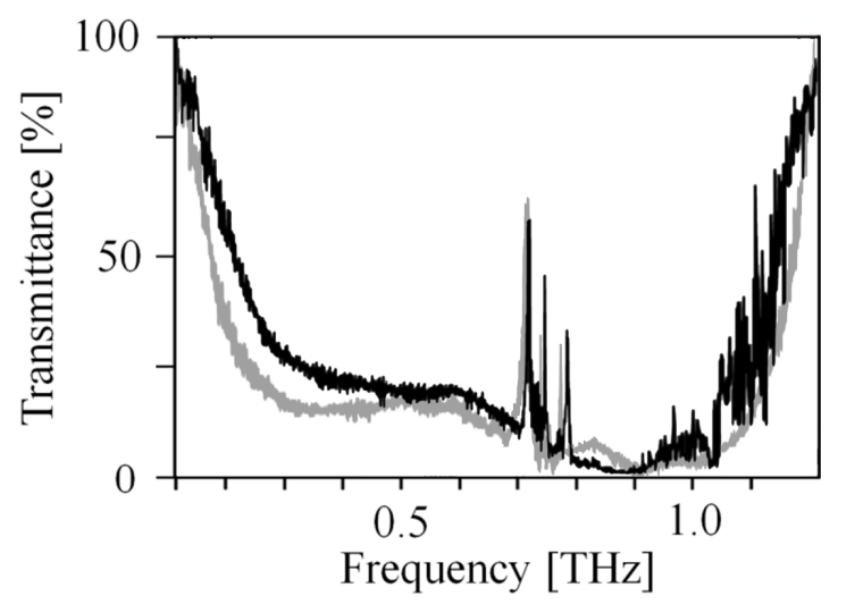

\section{Conclusions}

Micrometer order diamond lattices composed of acrylic resin with based metallic glass and oxide glass particles dispersion could be fabricated by using a stereolithographic technique through careful optimizations of process parameters. Magnetophotonic crystals were obtained through dewaxing and sintering heat treatments under the crystal transition temperature of metallic glass and above the melting temperature of oxide glass. The diamond structure could be exhibit a forbidden gap in a transmission spectrum of terahertz waves through Bragg diffraction. By introduction of an open hole as structural defect, localized modes of transmission peaks could be formed in the electromagnetic band gap according to the cavity size.

\section{Acknowledgments}

The authors would like to thank Hidemi Kato (Institute for Materials Research, Tohoku University, Japan) for providing metallic glass particle samples. 


\section{References}

1. Yablonobitch, E. Inhibited spontaneous emission in solid-state physics and electronics. Phys. Rev. Lett. 1987, 58, 2059-2062.

2. Inoue, M. Magnetophotonic crystals. J. Phys. 2006, 39, R151-R161.

3. Kirihara, S.; Takinami, Y.; Tasaki, S. Fabrication of metallodielectric photonic crystals to exhibit perfect millimeter wave band gaps. Int. J. Appl. Ceram. Technol. 2012, 9, 893-901.

4. Kirihara, S.; Kaneko, M.; Niki, T. Terahertz wave control using ceramic photonic crystals with a diamond structure including plane defects fabricated by microstereolithography. Int. J. Appl. Ceram. Technol. 2009, 6, 41-44.

5. Chen, W.; Kirihara, S.; Miyamoto, Y. Three-dimensional microphotonic crystals of $\mathrm{ZrO}_{2}$ toughened $\mathrm{Al}_{2} \mathrm{O}_{3}$ for Terahertz wave applications. Appl. Phys. Lett. 2007, 91, 153507-153509.

6. Angel, S.M.; Kulp, T.J.; Vess, T.M. Remote-raman spectroscopy at intermediate ranges using low-power cw lasers. Appl. Spectrosc. 1992, 46, 1085-1091.

7. Wang, H.; Wang, Q. Spectrum characteristics of nitrofen by terahertz time-domai spectroscopy. Phys. Conf. Ser. 2011, 276, 012209-012214.

8. Kutteruf, M.R.; Brown, C.M.; Iwaki, L.K.; Campbell, M.B.; Korter, T.M.; Heilwail, E.J. Terahertz spectroscopy of short-chain polypeptides. Chem. Phys. Lett. 2003, 375, 337-343.

9. Makino, A. New Functional Materials, Fundamentals of Metallic Glasses and their Applications to Industry; Inoue, A., Ed.; Technosystem: Tokyo, Japan, 2009; pp. 322-345.

(C) 2013 by the authors; licensee MDPI, Basel, Switzerland. This article is an open access article distributed under the terms and conditions of the Creative Commons Attribution license (http://creativecommons.org/licenses/by/3.0/). 